\title{
Putting psychology to the test: Rethinking model evaluation through benchmarking and prediction
}

\author{
Roberta Rocca ${ }^{1 *}$ and Tal Yarkoni ${ }^{1}$ \\ ${ }^{1}$ Department of Psychology, University of Texas at Austin \\ *Email: rbrrcc@gmail.com
}

Consensus on standards for evaluating models and theories is an integral part of every science. Nonetheless, in psychology, relatively little focus has been placed on defining reliable communal metrics to assess model performance. Evaluation practices are often idiosyncratic, and are affected by a number of shortcomings (e.g., failure to assess models' ability to generalize to unseen data) that make it difficult to discriminate between good and bad models. Drawing inspiration from fields like machine learning and statistical genetics, we argue in favor of introducing common benchmarks as a means of overcoming the lack of reliable model evaluation criteria currently observed in psychology. We discuss a number of principles benchmarks should satisfy to achieve maximal utility; identify concrete steps the community could take to promote the development of such benchmarks; and address a number of potential pitfalls and concerns that may arise in the course of implementation. We argue that reaching consensus on common evaluation benchmarks will foster cumulative progress in psychology, and encourage researchers to place heavier emphasis on the practical utility of scientific models.

Keywords: psychology, model evaluation, benchmarking, machine learning

The adherence to common standards for evaluating models and theories is an integral part of every science. A community of scientists that can find no agreement on what kind of work advances or hinders its goals is likely to experience difficulty making progress. A certain degree of flexibility in evaluation is, of course, both unavoidable and desirable: researchers in every field regularly disagree with one another about the importance and implications of specific findings, and such differences of opinion are widely understood to be an important catalyst of theoretical and experimental advances. But even the most contentious scientific disagreements typically unfold against the backdrop of some shared understanding of what is and isn't valid science: astronomers do not tend to argue with astrologists in academic journals, nor do molecular biologists battle with creationists.

Psychology is, in many ways, a field with a healthy respect for consensus and convention. One need only peruse the pages of most psychology journals to observe a remarkable degree of convergence in method: researchers tend to rely on similar patterns of reasoning, use similar statistical techniques, and even appeal to similar inferential quantities and binary thresholds (e.g., $\mathrm{p}<.05)$ to make critical decisions. Yet this convergence is, we will argue in this article, deceptive in key respects: beneath the superficial adherence to a common set of methodological conventions lies a realm of nearly unbounded evaluative freedom - one that imposes surprisingly few constraints on what researchers can or cannot do before they conclude that their preferred model is or is not supported by the available data.

Our argument is structured as follows. We begin by reviewing common evaluation practices in psychology and highlighting a number of features that have underappreciated, but severe, implications for our collective rate of progress. We then draw a contrast with evaluation practices in the field of machine learning, where researchers place far greater emphasis on common benchmarks and out-of-sample, practically meaningful predictions. We argue that many areas of psychology would benefit considerably from the adoption of such practices, and review a number of key desiderata for their successful implementation. We then discuss a number of examples within psychology where consensus benchmarks are either already in use, or have relatively clear paths to development and application. 
Finally, we consider common pitfalls and potential objections to the practices we have described. Our overarching conclusion is that convergence on consensus metrics and real-world benchmarks would advance the pursuit of traditional psychology goals (e.g., cumulatively building generalizable and reproducible knowledge), while simultaneously encouraging a focus on other highly desirable but historically underappreciated goals, such as maximizing the practical utility of scientific models and explanations.

\section{Model evaluation in psychology}

Empirical sciences seek to develop theories and models that accurately describe and predict relations between empirical phenomena. In a seminal paper, Breiman (2001) identified two main approaches to the problem of theory generation and validation. In the data modeling approach, researchers aim to develop models that reconstruct or approximate the true generative process underlying the data. In the algorithmic modeling approach, researchers proceed with few assumptions or ambitions with respect to the data-generating process; models are evaluated on their ability to produce desired outcomes (typically quantitative predictions), regardless of how they achieve that. The divide between data modeling and algorithmic modeling is paralleled by a distinction between explanation- and prediction-oriented model evaluation (Shmueli, 2010). In the explanatory tradition, models are evaluated favorably if they are comprehensible to researchers and display desirable qualitative features (e.g., the ability to capture specific patterns of effects). In prediction-oriented approaches, models are typically evaluated based on their generalization performance at predictive tasks - that is, the ability to approximate or correctly label new observations drawn from the same or similar populations the models were trained on.

Traditionally, the overwhelming majority of scientific practice in psychology has fallen within the data modeling/explanatory tradition. Theories are typically developed into hypotheses consisting of qualitative predictions about the directionality or magnitude of specific effects - that is, hypotheses about how parameter estimates for predictors of interest will deviate from the values expected under a null hypothesis. These qualitative predictions are often corroborated or disconfirmed via quantitative procedures, and indicators of statistical significance are often complemented with metrics of model performance that quantify models' ability to explain variance in the specific data sample.

The tendency to place heavier emphasis on qualitative effect patterns than on predictive accuracy is understandable in contexts where, as it is often the case in psychology, one's primary goal is to understand the internal structures that give rise to various aspects of mental function; merely demonstrating that a predictive model can quantitatively approximate specific behaviors provides no guarantee that it is capitalizing on similar processes as the human mind, let alone that its internal structure can be readily understood. Nevertheless, traditional model evaluation approaches in psychology suffer from a number of important weaknesses that threaten to undermine their ability to reliably assess how well a model is doing - even with respect to purely explanatory goals.

A first problem is that the ability of a model to display some desired qualitative pattern - e.g., statistical significance, or a good model fit-provides no guarantee that the model can actually do anything predictively useful. This is true even within the narrow context in which the effect was originally demonstrated: a model can produce highly significant effects of interest that nevertheless do not enable an analyst to non-trivially predict outcomes for new observations even when those observations are sampled from the same data-generating process as previously seen observations.

This problem is widely acknowledged in the literature, and it is often framed in terms of effect sizes - i.e., that significance isn't per se a guarantee of predictive validity; effect size also matters (Cumming, 2013; Kline, 2013; Nakagawa \& Cuthill, 2007; Sullivan \& Feinn, 2012). But effect size-based evaluation approaches also have important limitations, and can be contrasted with a predictive perspective that often reveals otherwise unavailable insights. 
To illustrate, we reanalyzed data from the Many Labs Project (Klein et al., 2014) - a large-scale multisite replication project investigating the replicability of 13 classical psychology effects in large samples collected across multiple independent sites ${ }^{1}$. The authors reported that 10 effects replicated consistently, with aggregate $p$-values below .001. However, the magnitude of these 10 effects varied wildly (with Cohen's $d$ ranging from 0.12 to 2.42). From a predictive standpoint, it is easy to demonstrate that even highly significant effects can produce essentially worthless predictions.

Consider two of the effects that putatively replicated consistently. First, take one of the anchoring effects (Jacowitz \& Kahneman, 1995), where participants are asked to provide an estimate of the height of Mount Everest after being exposed to unrealistically high or low anchor values. A simple least-squares regression model predicting the outcome variable (the estimated height of Mount Everest) from only the experimental condition assignment explains $57 \%$ of the variance. However, this estimate is, in principle, susceptible to overfitting (for discussion, see Yarkoni \& Westfall, 2017), because the same data is being used to fit the model and to evaluate its performance - in effect, double dipping. What happens when we reanalyze the data using a cross-validation procedure that estimates the out-of-sample predictive performance, using different subsets of the data to train and test the model? In this case, the answer is: not much. The estimated out-of-sample $R^{2}$ is still 0.57 -implying that our trained regression model predicts outcomes for entirely new individuals drawn from the same distribution just as accurately as outcomes for individuals it has already seen.

By contrast, if we repeat the same analysis on the "sunk cost" effect in Many Labs - where participants are asked to report how likely they would be to go see a football game on a freezing cold day, assuming that they either paid for their ticket or were given it for free - we get a very different picture. Here, the in-sample $R^{2}$ is an already modest 0.02 , but the out-

\footnotetext{
${ }^{1}$ The code for these analyses is available on GitHub at (https://github.com/rbroc/benchmarks_paper).
}

of-sample $R^{2}$ is actually negative $(-0.02)^{2}$. In other words, it's not just that the effect is "statistically significant, but small", as a traditional interpretation might suggest; the model is actually completely incapable of using knowledge of the experimental assignment to improve its prediction of game attendance in new individuals. Worse still, the above analysis is arguably something of a best-case scenario, because the large training sample naturally mitigates overfitting (we return to this point later). If we fit separate models for each experimental site (i.e. for sample sizes varying between 79 and 1323 participants), we observe a $p$-value below .05 for 15 out of 36 sites. For all but one of these sites, the out-of-sample $R^{2}$ is negative, but the in-sample $R^{2}$ ranges up to 0.08 (for $\mathrm{n}=174$; see Figure 1). In this scenario-which is arguably much closer to the modal psychology study in terms of sample size and reported $p$-value - we might draw radically different conclusions about the importance or utility of our model if we failed to consider its true (i.e., out-of-sample) predictive capacity.

Given this finding, should one still count the "consistent replication" of the sunk cost effect reported in Many Labs (as expressed by $p$-values) as support for any theoretical hypothesis? Maybe - but it is far from obvious. And similar problems will often go unnoticed when using conventional evaluation procedures. Researchers who focus exclusively on in-sample statistics might never realize that a statistical model they interpret as support for some psychological hypothesis may be incapable of generating any meaningful prediction even when applied to new data sampled from exactly the same distribution, let alone to data obtained from a different measurement context.

A second problem is that many of the procedures psychologists traditionally use to evaluate their theories and models are widely misunderstood, and consequently, misinterpretation of results is common. It

\footnotetext{
${ }^{2}$ Some readers may question this value, under the belief that $R^{2}$ values must have a lower bound of 0 . But under the traditional definition of $R^{2}$ (i.e., 1 minus the ratio of residual to total variance), it is possible to have an arbitrarily large negative $R^{2}$. Values below 0 imply that the model performs worse than a constant model that simply uses the sample mean as the prediction for all individual observations.
} 


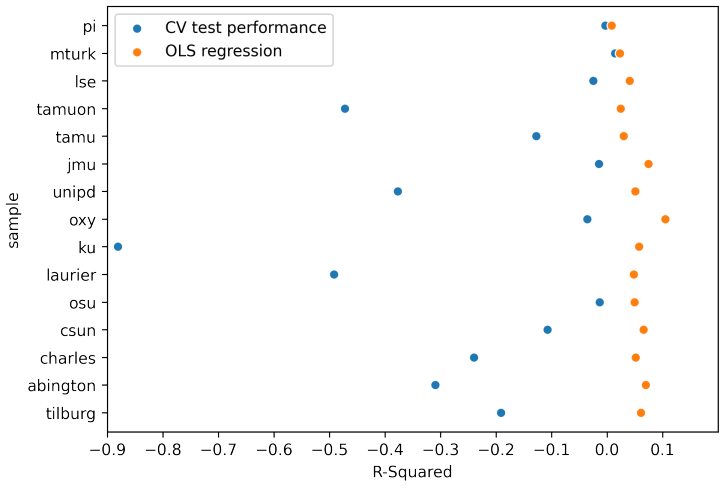

Figure 1: In-sample $R^{2}$ and out-of-sample $R^{2}$ (from 10 -fold cross-validation) for sunk cost effects from (Klein et al., 2014), for each experimental site where the effect reaches statistical significance $(\mathrm{p}<.05)$. For many sites, in-sample and out-of-sample $R^{2}$ differ widely due to overfitting. Inflated in-sample $R^{2}$ can lead to overly optimistic interpretations of the true predictive capacity of a model when out-of-sample performance is disregarded.

has been observed for decades, for example, that most psychologists do not appear to understand the meaning of the $p$-values they so avidly use as decision aids (Gelman, 2013; Greenland, 2011; McShane, Gal, Gelman, Robert, \& Tackett, 2019). The coefficient of determination $\left(R^{2}\right)$ is frequently interpreted as a general measure of a model's predictive capacity, yet it does not, by itself, indicate whether a model is capturing relevant and generalizable patterns in the data, or is merely overfitting to spurious error sources (e.g., measurement error; J. I. Myung \& Pitt, 2018). A whole host of inferential mistakes routinely arise when researchers draw unsupported causal inferences from observational data using standard regression methods - e.g., treating regression coefficients as causal estimates without disclosing and defending causal assumptions (Rohrer, 2018; Watts, 2014; Watts et al., n.d.), or concluding that a construct of interest has incremental validity over and above other constructs simply because a given measure of that construct makes a statistically significant contribution after "controlling for" putative confounds
(Kahneman, 1965; Shear \& Zumbo, 2013; Westfall \& Yarkoni, 2016).

A third weakness of standard evaluation practices is their marked idiosyncrasy. The choice of empirical and statistical operationalization of a given hypothesis, as well as the concrete implementation of the analysis workflow, is typically left almost entirely to the discretion of researchers' individual preferences. And researchers freely exercise that privilege. If theories in psychology are like toothbrushes, in that "everyone has one, and nobody wants to use anyone else's" (Mischel, 2008), operationalizations of theories are, perhaps, like pieces of chewing gum: most researchers do not seem to want to use even their own a second time.

We are not suggesting that this norm is unjustified; there are often excellent reasons why a particular theory or model cannot be rigorously tested without introducing new experimental designs or methodological procedures to the literature. But the practice clearly also has heavy costs. Chief among these is a lack of standardization, which severely limits the commensurability of different studies - even those nominally seeking to test competing theories of the same phenomenon. When two psychologists disagree about the merits of their respective theories, it can be difficult to determine where the disagreement actually resides. Are there competing interpretations of the same data point? Is the disagreement over whether a given experiment is a good test of a theory? Do the theories actually differ in scope, despite using similar nomenclature?

Absent consensus benchmarks that encourage commensurability and provide at least a partial basis for objective evaluation of different researchers' output, there is little to prevent people from adopting whichever procedures, and emphasizing whichever results, they find most expedient. It can be difficult for a field to make collective progress when, to a significant extent, each researcher gets to decide for herself, using their own idiosyncratic evaluation criteria, how well they are doing. The fact that researchers overwhelmingly justify their evaluations using similar quantitative criteria is of little help: it does one little 
good to know that $90 \%$ of the researchers in a field agree that a $p$-value below .05 means one's hypothesis is corroborated so long as each researcher gets to freely define the hypotheses, measurement context, and statistical model that produce that $p$-value.

In highlighting these weaknesses, we are not trying to add fuel to the long-burning fire of statistical criticism aimed at psychologists. What is salient to the present argument is not so much the observation that psychologists frequently engage in suboptimal evaluation practices; it is that extended contact with empirical data rarely seems to provide much corrective feedback. What does it imply about the reliability of a discipline's output if a large fraction of its practitioners can spend their careers "successfully" using standard evaluation procedures without reality ever intruding onto, and correcting, fundamental misconceptions about those same procedures? When civil engineers screw up their calculations, bridges fall down; when physicists make basic mathematical errors, those mistakes ramify quickly to the point of detection and correction; even a new driver who fails to maintain sufficient distance from the cars in front of them is likely to learn some caution the hard way. Yet in most areas of psychology, a researcher can spend years happily using $p$-values to decide whether theories should live or die, and still come away confidently asserting that a $p$-value below 0.05 means there is a $95 \%$ probability the hypothesis is correct. If standard evaluation procedures in psychology cannot be trusted even to identify and rectify glaring misunderstandings about those procedures themselves, how can they be trusted to assess the quality of psychological theories?

These fundamental issues highlight the need to rethink evaluation practices in favor of more reliable consensus procedures, where models are regularly submitted to solid "reality checks". To achieve this, researchers may be better off drawing inspiration from prediction-focused fields like machine learning, where there is a strong emphasis on evaluating model performance using consensus datasets, out-of-sample prediction metrics, and benchmarks that have real-world implications. In the next sections, we will review a number of these practices and discuss ways psychology can learn from some of the strengths of the machine learning model (we will also discuss some of the liabilities later).

\section{Model evaluation in machine learning}

The practice of training and evaluating models on public benchmarks is well-entrenched in machine learning research. One reason for this is the field's long track record in building large datasets. Developing datasets of size suitable to efficiently train machine learning models - and particularly highly parameterized deep neural networks - has historically been resource-intensive and time-consuming, which has encouraged researchers to coordinate data collection efforts and publicly share the resulting resources. As a consequence, the presence of consensus datasets has naturally provided to the machine learning community a set of reference empirical problems on which models can be evaluated and compared.

Some milestone datasets have proven especially central to the development of the field, directly triggering the impressive advances observed, for example, in computer vision and natural language processing over the last decades. The most popular and perhaps most influential example is ImageNet (Deng et al., 2009), a massive, continuously updated, database of labeled images of objects built on the WordNet ontology (Miller, Beckwith, Fellbaum, Gross, \& Miller, 1990). At present, ImageNet includes around 14 million images from more than 20,000 object categories, and it is still widely used to train and evaluate computer vision models. Crucially, the release of ImageNet was followed by the launch of the annual ImageNet Large Scale Visual Recognition Challenge (ILSVRC), in which machine learning researchers competed to develop models able to perform well on a 1000-class image classification problem. It was in the attempt to solve the ImageNet challenge that the AlexNet model (Krizhevsky, Sutskever, \& Hinton, 2012) - often pinpointed as a critical milestone in the "deep learning revolution" (LeCun, Bengio, \& Hinton, 2015) - emerged (Figure 2).

The ImageNet case is a textbook example of how cleverly designed benchmarks posing challenging predictive problems can boost progress in research 
targeting key long-standing problems. But the introduction of new benchmarks can also contribute to shifting the focus towards less developed research topics. One example of this is the rising interest in the problem of grounded language learning within natural language processing. Most traditional language models are trained exclusively on text: models learn high-dimensional linguistic representations from statistical patterns in massive corpora (e.g. CommonCrawl), a strategy which has proven effective for many applications of artificial language systems. State-of-the-art transformer models (Devlin, Chang, Lee, \& Toutanova, 2018; Vaswani et al., 2017) achieve previously unthinkable levels of performance even on challenging tasks such as question-answering and summarization. However, many instances of real-world language rely on joint processing of linguistic input and sensory and motor information (e.g., spatial navigation requires linking words to visual input and actions),

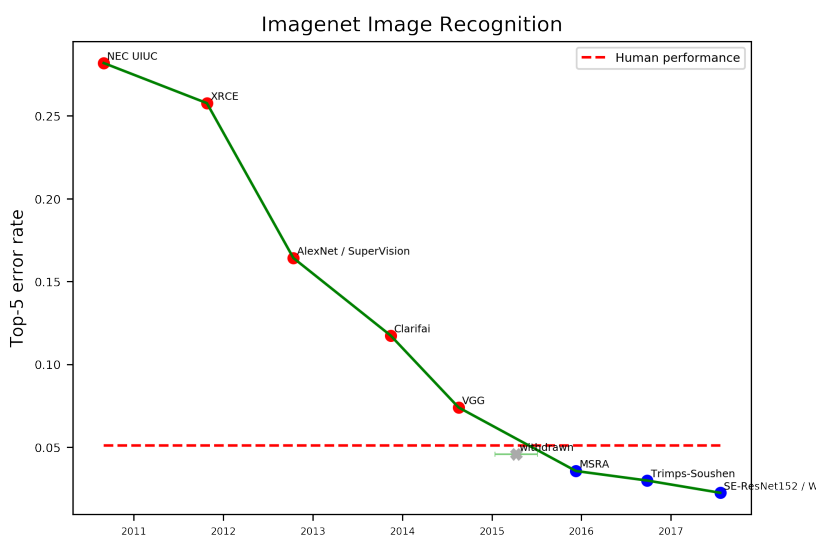

Figure 2: Performance of computer vision models on the ImageNet challenge over time (2010-2017). The release of ImageNet has strongly incentivized the development of increasingly more powerful computer vision architectures. AlexNet was a crucial breakthrough, achieving a 10 -percent drop in error rate compared to the previous models. Current state-ofthe-art models outperform humans. Retrieved from https://www.eff.org/ai/metrics. and most current language models lack direct access to non-linguistic inputs. The need to integrate sensorimotor knowledge into the semantic representations learned by language models has recently led to the development of a number of benchmarks tackling vision-and-language tasks (e.g. Touchdown: https://sites.google.com/view/streetlearn/touchdown, or Alfred: https://askforalfred.com/), aimed at encouraging the community to focus on this challenging subdomain (Chen, Kornblith, Norouzi, \& Hinton, 2020; Shridhar et al., 2020).

Another important consequence of the strong emphasis placed on benchmarks is that, over time, the repertoire of available benchmarks has expanded and diversified, which makes it possible, in many domains, to test model performance on a battery of tasks and quickly gain a comprehensive overview of its strengths and liabilities. Once again, natural language processing offers an excellent example: language models are generally trained on one or more general-purpose objectives (e.g. next-word prediction), and, after (often minimal) fine-tuning, they are evaluated against composite benchmarks (e.g., Sakaguchi, Le Bras, Bhagavatula, \& Choi, 2019; Wang et al., 2019). In this regard, a particularly interesting example is that of GPT-3 (T. B. Brown et al., 2020), a language model optimized for next-word prediction, but whose architecture is entirely task-agnostic. The model can thus be directly (i.e. without fine-tuning or gradient updates) evaluated on virtually any task that can be framed in terms of context-completion pairs using a learning framework, named few-shot learning, which mimics humans' learning by demonstration ((w. B. Brown et al., 2020). Using this framework, GPT-3 was evaluated on a wide range of both wellestablished benchmark tasks (e.g. pronoun resolution) and novel tasks (e.g. generating sentences which contain non-words). This simple but groundbreaking evaluation protocol has enabled researchers to quickly gain a comprehensive overview of GPT-3's strengths (e.g. in tasks such as modeling of long-range dependencies) and weaknesses (especially apparent in tasks such as common sense reasoning or arithmetic tasks), paving the way towards further improvements.

Finally, emphasis on benchmarks has also led to 
the development of standardized protocols for minimizing overfitting, which are now gaining popularity in many other fields. The theory and practice of crossvalidation and regularization methods (e.g., lasso and ridge regression for linear models, or dropout for neural networks), was largely developed in statistics and machine learning (though it is intriguing to note that some of these methods have their initial roots in psychometrics - e.g. Mosier, 1951), alongside practices that aim to minimize overfitting during model selection and pipeline construction (e.g., the custom of holding out test datasets in predictive modeling competitions).

\section{Towards useful metrics and bench- marks}

Machine learning's pervasive reliance on objective performance benchmarks arguably follows quite naturally from its practitioners' tendency towards an algorithmic modeling philosophy. The situation in psychology is rather different. Most psychologists have thus far demonstrated little inclination towards adoption of common benchmarks, and active efforts may be needed to successfully introduce many of the beneficial practices and conventions currently found in the ML community. In this section, we discuss a number of core principles that would maximize the utility of benchmarks in psychology, and identify concrete steps researchers can take to help realize them. We hasten to emphasize that these principles are neither necessary nor sufficient for success, and are likely to vary widely in importance across different areas of research.

\section{Large datasets}

As the case of machine learning shows, identifying or developing suitable datasets is often the first and most important step in developing good benchmarks. The most important criteria for a good benchmarking dataset are that it include large and highly variable samples on key dimensions of interest (e.g., participants, experimental paradigms, and stimulus sets, see Oberauer et al., 2018), and be publicly available and easily accessible. Two other characteristics are also desirable in many cases: data should be collected in settings reflecting naturalistic conditions, increasing the ecological validity of high-performing models; and datasets should lend themselves to predictive tasks with explicit practical utility.

In contrast to machine learning, psychology does not have a strong tradition of collaborative building and public sharing of large datasets (though we will discuss several exceptions later). The advent of the big data era has been welcomed by many psychologists as a potential game-changer, but its influence on research practices in most areas has thus far been minimal (Yarkoni \& Westfall, 2017). To a large extent, psychologists continue to prioritize small-batch, hand-crafted, artisanal carefully-tailored, small-sample, in-house experimental manipulations over large-scale analyses of general-purpose datasets produced by other researchers. Although there is much to be said for the ability to design experimental procedures that directly address one's chosen research question, many psychologists fail to fully appreciate the countervailing costs - i.e., the degree to which small samples inflate effect sizes (Gelman \& Weakliem, 2009; Ioannidis, 2008; Yarkoni, 2009) and (in the presence of selection bias) false positive rates (Button et al., 2013); are susceptible to statistical and procedural overfitting (Yarkoni \& Westfall, 2017); and contribute to publication bias (Ferguson \& Heene, 2012; Open Science Collaboration, 2015; Vasishth, Mertzen, Jäger, \& Gelman, 2018) and widespread generalization failures (Yarkoni, 2019).

Importantly, the acquisition of large datasets does not have to impose a huge burden on individual researchers. An effective strategy is to coordinate data collection across research groups and publicly share the resulting datasets. Large-scale efforts of this kind have already been conducted in psychology and neuroscience. Notable examples are the Psychological Science Accelerator (https://psysciacc.org/, see Moshontz et al., 2018), the ManyLabs (Klein et al., 2014) and ManyBabies (Frank et al., 2017) replication projects, the Human Connectome Project (Van Essen et al., 2013), BioBank (Allen, Sudlow, Peakman, Collins, \& UK Biobank, 2014), lexical datasets such as the English Lexicon Project (Balota 
et al., 2007) and the Small World of Words dataset (De Deyne, Navarro, Perfors, Brysbaert, \& Storms, 2019; De Deyne, Perfors, \& Navarro, 2016), large neuroimaging datasets such as the Naturalistic Neuroimaging Database (Aliko, Huang, Gheorghiu, Meliss, \& Skipper, 2020), the Narratives database (Nastase et al., 2019) and datasets from the Courtois project on neuronal modeling (CNeuroMod, https://www.cneuromod.ca/), or the neuroimaging and genomics dataset ENIGMA (http://enigma.ini.usc.edu/). Social science datasets such as the General Social Survey (https://gss.norc.org/), the World Values Survey (http://www.worldvaluessurvey.org), the World Inequality Database (https://wid.world/) and the American National Election Studies (https://electionstudies.org/) are also relevant to many areas of psychology research. Moreover, online data collection platforms like Amazon Turk (https://www.mturk.com/) or Prolific (https://www.prolific.co/), as well as relatively novel data types such as social media data and mobile device sensors data, often make development of large datasets even less effortful and resource-intensive. Conditions are thus favorable to initiate and promote the creation of consortia that would harness these resources on a large scale.

Compiling lists of available resources would be a good practical first step. In some cases, datasets may already exist that afford interesting predictive challenges. These resources could be easily repurposed, and their use as benchmarks promoted via data papers that describe their characteristics and provide baseline models (Chavan \& Penev, 2011; K. Gorgolewski, Margulies, \& Milham, 2013).

\section{Good target measures and performance metrics}

Development of good benchmarks relies on the choice of good target measures and of performance metrics that adequately reflect a model's predictive validity. To avoid some of the main downsides of commonly used metrics and ensure reliable evaluation, benchmarks should thus meet at least two important criteria.

First, target variables included in benchmark datasets should take seriously traditional psychometric considerations like measurement reliability and construct validity. That is, a good target measure should primarily measure the phenomenon it is intended to measure, and have minimal contribution from other constructs or methodological confounds. So stated, this point may seem obvious, but the history of psychology suggests it is far from trivial. In many domains of psychology, core constructs are routinely operationalized in highly idiosyncratic ways (Eisenberg et al., 2019), and logical fallacies in the operationalization of constructs are pervasive (Cronbach \& Meehl, 1955; Meehl, 1978, 1990). Outside of a few sub-fields (e.g., personality and individual differences, psychopathology, and industrial-organizational psychology), reliability and validity are rarely systematically assessed. While good psychometric properties are desirable in any study, they are particularly critical when developing performance benchmarks likely to be used by other researchers, as any biases or errors risk being widely (and usually silently) propagated. Identifying good evaluation metrics will thus require research communities to engage in constructive debate informed by thorough (logical and empirical) assessments of the validity of each candidate measure.

Second, the quantitative metrics used to evaluate model performance also need to mean what researchers think they mean, and should be appropriately tailored to the intended use case(s). As discussed earlier, one way in which evaluation metrics in psychology commonly fail to meet this criterion is by relying on in-sample rather than out-of-sample performance estimates. For example, $R^{2}$ - probably the most widely used evaluation metric in psychology - is typically reported for the very same sample used to fit the model. This approach fails to distinguish signal from noise, and will generally produce inflated estimates of a model's true capabilities (Hofman, Sharma, \& Watts, 2017; Mahmoodi, Leckelt, van Zalk, Geukes, \& Back, 2017; Shmueli, 2010; Yarkoni \& Westfall, 2017). Using cross-validation provides a simple but effective guard against these issues (see Figure 3 ).

A related problem is the selection of a metric illsuited to its intended downstream applications. For example, $R^{2}$ is a relative measure of fit (i.e. its in- 


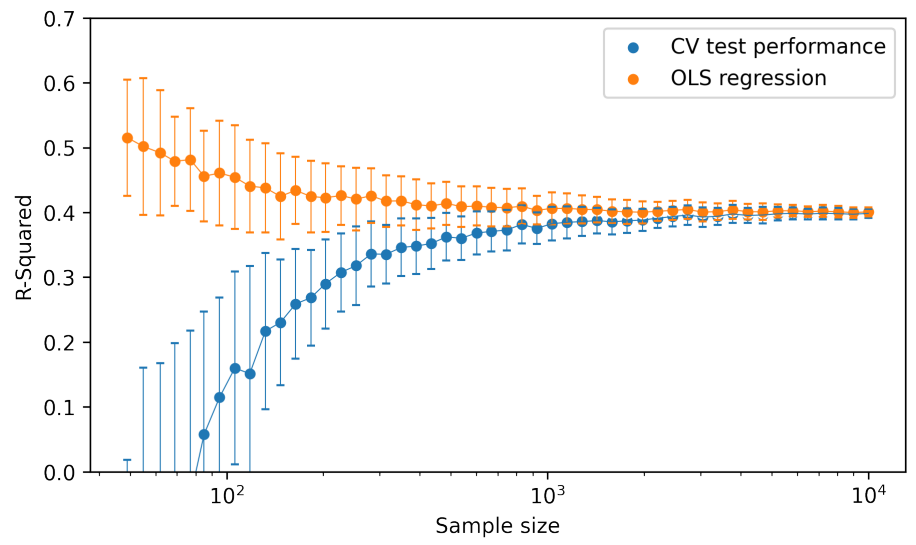

Figure 3: In-sample $R^{2}$ from OLS regression and average test $R^{2}$ in a 10-fold cross-validated regression on simulated data. Data are drawn from an 11-dimensional multivariate normal distribution (10 used as predictors, one as outcome). All dimensions have zero mean and unit variance. Predictors are orthogonal and each correlates .2 with the outcome variable. We systematically varied the number of samples drawn (x-axis), and repeated sampling and model fitting 100 times per sample size. Error bars represent standard deviation over repeated iterations. For small sample sizes, in-sample performance from OLS regression returns an overly optimistic estimate of the predictive accuracy and generalizability of the model (due to overfitting), while out-of-sample CV accuracy returns a more accurate picture of its actual predictive performance. In addition to mitigating overfitting, cross-validation procedures offer the additional advantages of being relatively straightforward to implement and "model agnostic"; they can be applied to virtually any model-fitting framework, enabling direct comparison of any set of models.

terpretation depends on the total variance within a specific sample), and it provides no explicit information about the overall accuracy of individual predictions - in contrast to absolute metrics such as mean squared error or mean absolute error, which allow interpretation of the error associated with individual predictions. In many applications where psychologists routinely use relative metrics (e.g., quantifying variance explained in psychopathology symptoms), absolute metrics would arguably be more appropriate and more informative with respect to the actual utility of the model for applied predictive purposes.

\section{Clear practical implications}

A large proportion of research in psychology only indirectly affords a direct translation into prediction/explanation of real-world phenomena. Ideally, benchmarks should help counter this tendency by devising predictive tasks grounded in real-world challenges, and that have clear practical implications. Such an emphasis is desirable for several reasons. First, it facilitates consensus evaluation of the merits of a theory or model, as predictions or key results will often be rendered in intrinsically meaningful quantities (e.g., overt ability to discriminate patients from controls, rather than goodness-of-fit of a particular latent variable model). Second, models that demonstrably perform well on relatively naturalistic tasks are more likely to successfully generalize to real-world use, as there will typically be fewer points of potential failure between model evaluation and model application. Third, the availability of models with clear real-world implications is likely to attract increased interest in, and funding for, psychology research.

Naturally, the extent to which practical applicability can or should be considered central to benchmark 
tasks will vary widely across sub-fields and research questions. Industrial and organizational psychology is a good example of a field where prediction of practical outcomes (e.g. organizational success) on the basis of variables that can be intervened upon (e.g. social network structure within the organization) is a foundational goal. Large-scale studies have been conducted targeting applied challenges such as predicting team effectiveness from wearable sensor data capturing team interactions and communication patterns (Kozlowski \& Chao, 2018), or using text mining to identify issues with customer satisfaction (see Guzzo, Fink, King, Tonidandel, \& Landis, 2015 for an overview of big data studies in I/O psychology) In this field, developing benchmarks with an applied angle would merely require coordination and scaling of existing resources.

Predicting real-life outcomes is also an important goal in educational psychology (where understanding the factors that impact educational achievement can directly inform teaching strategies and education policies), in political psychology (where large-scale surveys aimed at informing prediction of voting behavior are periodically conducted, and many efforts are devoted to developing text models detecting toxic or misleading political contents), and in several domains of clinical research (where predictive models are used for purposes ranging from causal modeling of psychiatric diagnoses, to predicting patients' risk of being subjected to mechanical restraint, see Danielsen, Fenger, Østergaard, Nielbo, \& Mors, 2019). Other clinical fields have fewer readily available datasets, but also have high potential. For example, brain stimulation datasets could be used to develop models yielding individualized predictions of the boundaries of specific brain circuits (e.g. language areas), with direct applications for fMRI-based presurgical mapping (Benjamin et al., 2017; Bookheimer, 2007). In this and other cases, efforts to develop relevant resources could significantly promote progress in solving challenges of high practical significance.

\section{Data sharing infrastructure and open source software}

Large datasets, solid metrics and an applied focus are important foundations of good benchmarks, but their utility is often limited by the absence of suitable infrastructure and tools. Ideally, good benchmarks should be released alongside resources that enable users to readily access and manipulate benchmark datasets; train, test, and deploy models; and publicly share validation results. The availability of highquality and domain-general infrastructure for storage, sharing and querying of large datasets is currently limited in psychology, though the situation is rapidly changing for the better. For data storage and sharing, the Open Science Framework (OSF; https://osf.io) is the most widely used platform. OSF provides storage and sharing capacity for a wide variety of data and resources (not limited to psychology). However, it comes with no data sharing standards, and resources to programmatically access OSF material are still limited. The neighboring domain of neuroscience is an excellent source of inspiration on how to overcome these challenges. An integrated suite of tools which includes a platform for data sharing (OpenNeuro, see K. Gorgolewski, Esteban, Schaefer, Wandell, \& Poldrack, 2017), data sharing standard ( $B I D S$, see K. J. Gorgolewski et al., 2016) and tools for programmatic access of BIDS data such as PyBIDS (Yarkoni et al., 2019) has been developed over the last few years. There are promising signs that other areas of psychology are moving to adopt a similar model-e.g., there is an incipient effort to develop a lightweight, BIDS-like specification for representing psychological dataset (Psych-DS; https://psych-ds.github.io/).

Complementary to improvements on the datasharing side, high-level tools should be developed that enable easy access to benchmark datasets. A potential source of inspiration is the $n l p$ library developed by HuggingFace, which enables easy programmatic access to popular datasets for natural language processing. Furthermore, software facilitating training and deployment of models on reference datasets would be highly beneficial. Examples of similar tools (coming, again, from natural language processing) are libraries such as HuggingFace's transformers, or broader-scope ones 
such as spaCy and AllenNLP (Gardner et al., 2018). These libraries offer functional routes and pipelines to easily train, fine-tune, evaluate and deploy models, making NLP tools accessible also beyond the narrow community of specialists. Availability of similar packages for psychology would also make datasets and models readily available as a "service" for and beyond the research community, and encourage their deployment in practical (e.g. societal or technological) applications. Such tools could also be conveniently paired with packages for model diagnostics, checking for basic validity criteria such as falsifiability or identifiability (I. J. Myung \& Pitt, 2002; Oberauer et al., 2018), or performing post-hoc tests on model predictions to identify biases.

As the example of the BIDS ecosystem in neuroimaging attests, organizing recurring events (workshops, conferences, hackathons) and setting up consortia dedicated to defining the structure of these tools could be an effective way to promote their development. Openness, integration and communitybased development should be core principles of this endeavor.

\section{Well-aligned incentives}

Most of the benefits of the benchmarking practices we have described apply at the level of entire research communities. But to attain widespread adoption, such practices also need to be incentivized at the individual level. Here, machine learning again offers a source of inspiration. In machine learning, it is common practice to release benchmarks in combination with competitions that encourage the development of systems that can advance the state of the art on the target task. This competition-oriented culture is perhaps inherited from artificial intelligence, whose development has been profoundly shaped by the goal of outperforming humans on challenging intelligence tasks (notable examples are the chess match between Garry Kasparov and IBM's Deep Blue chess computer, or the Go match between Lee Sedol and DeepMind's AlphaGo model), and it has been fostered by milestone events such as the 2006 Netflix Prize Competition (Bennett, Lanning, \& Others, 2007) and the already mentioned ImageNet Large Scale Visual Recognition
Challenge (ILSVRC).

Over the years, predictive challenges have become increasingly popular beyond machine learning. This competition-oriented and community-based model development framework is today prominently embodied by Kaggle (https://www.kaggle.com/competitions), a platform hosting and promoting data science challenges, which simultaneously advances funders' interests, encourages technical progress via communitydriven efforts (Garcia Martinez \& Walton, 2014), and provides an important training grounds for both budding and expert data scientists. Contests and competitions are also gaining momentum in cognitive neuroscience, a field that places increasing emphasis on data sharing resources and tools for reproducible data (re)analysis. A notable example is the ongoing TReNDS Neuroimaging Kaggle competition (https://www.kaggle.com/c/trends-assessmentprediction), where the goal is to develop models that robustly predict multiple normative assessments from multimodal MRI features.

Promoting contests and competitions is one of the measures that could prove effective in encouraging researchers to adopt benchmarking practices on a large scale, but a complementary system of incentives needs to be put in place in publication. Clear and detailed model descriptions, and (wherever possible) reporting of predictive performance on benchmark tasks should be demanded when reviewing papers making quantitative claims. Publication formats tailored to dataset, model or metrics development may be developed to incentivize scientists to invest more time and resources in this type of work.

\section{Adaptiveness}

Good benchmarks dynamically adapt over time to the goals and developments of a field: they should evolve and improve, with new benchmarks being introduced as the field evolves, and old benchmarks occasionally becoming obsolete when performance plateaus or when their intrinsic limitations become apparent. Machine learning offers an interesting example of the latter scenario - one that illustrates how a heavy emphasis on benchmarking and consensus 
metrics can often encourage methodological debate rather than silencing it.

The example comes from computer vision, where it has been noted that the majority of models that achieve near-perfect performance on ImageNet classification tend to perform very poorly in real-world applications (Barbu et al., 2019). Low reliability of benchmark metrics in reflecting models' predictive performance in real-world tasks has been attributed to limitations intrinsic to the ImageNet dataset: the images only display objects as seen from a limited set of viewpoints, and on a limited set of background types. Systems trained on ImageNet may thus learn to identify objects based on dataset-specific features of images that are poorly correlated with the corresponding object classes in the real world, leading to poor generalization in naturalistic scenarios. These considerations have led to the development of a new benchmark, ObjectNet (Barbu et al., 2019), where features of the visual context such as viewpoint and background are systematically controlled for. Performance of traditional computer vision architectures on ObjectNet drops dramatically compared to ImageNet, which has readjusted beliefs on the capabilities of state-of-the-art models, and encourages the development of new models that can overcome these weaknesses.

Importantly, benchmarks should be continually evaluated and revised not only for performance-related reasons, but also for ethical ones. Benchmarks are introduced in specific historical and social contexts, and they may not be immune to severe biases. A critical re-evaluation of machine learning benchmarks over the last years has revealed the presence of racial and gender biases in datasets and models, boosting interest in fair AI (Zou \& Schiebinger, 2018) and highlighting the need for a radical rethinking of research practices and the structure of research communities (Kalluri, 2020). Here, too, dynamicity has proven a feature rather than a bug, encouraging ethical scientific practices.

Benchmarks that satisfy all the criteria outlined in this section can dramatically improve the current state of model evaluation in psychology, promote cumula- tive scientific progress, and help overcoming other major liabilities in research practices. Such benchmarks would not only provide objective and reliable criteria for "model-agnostic" (Ribeiro, Singh, \& Guestrin, 2016) assessment of model performance - i.e. evaluation criteria independent of the specific characteristics of and structural similarity between models; they would also encourage researchers to adopt more transparent research practices by formalizing their models and making their theoretical assumptions and operationalizations of target constructs explicit. However, the degree to which all these conditions can be fulfilled varies widely across fields.

\section{Existing work and feasibility across fields}

We have outlined some core principles that would facilitate the development of benchmarks that fit the overall characteristics and goals of psychology. Psychology, however, is a complex and multifaceted domain: its subfields vary in their methodological profile, in the importance attributed to prediction of real-world outcomes, and in the extent to which they are sensitive to the need for common metrics. As a consequence, some fields have already come close to developing evaluation benchmarks, while others still struggle to introduce consensus metrics and big data into standard workflows. In what follows, we will present examples of fields where practices favourable to the introduction of benchmarks are already established, and point the way to further developments. We will then provide examples of fields where such practices are less common, and discuss how existing resources could be leveraged to promote them.

\section{Qualitative benchmarks in working memory research}

One of the fields that has come closest to introducing evaluation benchmarks is that of short-term and working memory research, where development of benchmarks for model and theory evaluation has been explicitly advocated and undertaken in work by Oberauer et al. (2018). The authors ground their attempt to develop benchmarks for short-term and 
working memory research in the observation that the number of novel empirical findings in psychology has been growing so dramatically over the years that no psychological theory can realistically account for all of them. When testing their theories, researchers thus inevitably select a preferred subset of facts or even "manufacture" novel ones using new experimental paradigms. This results in most psychological theories having non-overlapping empirical scope, which makes objective comparison difficult or impossible. In the authors' view, introducing benchmarks would allow researchers to focus theory-development efforts on a set of substantive findings that any valid theory should account for, and to objectively compare models on their ability to do so.

The project undertaken by Oberauer and colleagues provides an excellent model for how to practically develop a set of consensus evaluation tasks and metrics collaboratively and transparently. Benchmark development was carried out by a consortium that gathered a team of scientists from a variety of research institutions and theoretical backgrounds, and their collaboration unfolded over a series of seminars and workshops. Updates on the project were progressively reported on the project's webpage (https://wmbenchmarks.wordpress.com), where a public forum also gathered input from the broad research community. The set of benchmarks developed over the course of the project is now publicly available on GitHub (https://github.com/oberauer/BenchmarksWM).

Our proposal aligns with this outstanding work on the motivation and need for benchmarks; however, there are a few differences in the proposed approach. First, in Oberauer and colleagues' proposal, benchmarks are designed to evaluate models on the extent to which their predictions globally capture solid and replicable statistical effects. We argue that models should ideally also be evaluated on their ability to quantitatively predict individual data points. Furthermore, in our view, benchmarks should privilege predictive challenges that focus on complex, naturalistic data, so as to favor models whose predictions generalize to real-world problems, rather than aiming at merely replicating well-established findings from controlled experimental settings. We recognize, of course, that the feasibility of this goal varies largely across fields.

\section{Genome-wide association studies and poly- genic scores: the case of statistical genetics}

A second field that offers useful parallels for psychology is statistical genetics. Research in statistical genetics focuses on clarifying to what extent, and how, phenotypic variation along complex behavioral traits (be it binary traits such as diagnoses, or quantitative traits such as personality dimensions) can be attributed to heritable genetic influences (Fisher, 1919; Wright, 1920) and various environment factors. Although we are not aware of efforts to explicitly introduce common benchmarks to this literature, many of the features we have advocated for are already embedded in standard workflows.

Multi-site data collection and data sharing have long been common in statistical genetics (Haworth, Davis, \& Plomin, 2013; Hur \& Craig, 2013). In earlier stages of the discipline, where techniques for direct, large-scale inspection of the genome were not yet available, heritability had to be estimated indirectly by means of twin and family studies (Plomin, DeFries, Knopik, \& Neiderhiser, 2016), which require gathering data from individuals in rather rare groups and configurations. Novel data collection and analysis techniques, however, have made large datasets and public data sharing even more necessary. One key development has been the introduction of genome-wide association studies (GWAS; see Visscher, Brown, McCarthy, \& Yang, 2012), which can support identification of specific genome-phenotype associations. As complex traits are highly polygenic (Chabris, Lee, Cesarini, Benjamin, \& Laibson, 2015), adequately powered GWAS require sample sizes on the order of at least tens of thousands (Dudbridge, 2013). This has provided even further incentives for the development of large-scale collaborations (e.g. the Wellcome Trust Case Control Consortium, https://www.wtccc.org.uk/) and infrastructure for effective data sharing (e.g. GWAS central, https://www.gwascentral.org/).

Findings from this line of work have dramatically 
reshaped beliefs about the complexity of the genetic mechanisms underlying most phenotypes, and have led researchers to reconsider many findings from candidate gene studies now deemed spurious (Border et al., 2019; Chabris et al., 2015; Duncan \& Keller, 2011). Importantly, GWAS has also facilitated the emergence of consensus on target metrics and promoted heavier emphasis on prediction (de Los Campos, Sorensen, \& Gianola, 2015; de Los Campos, Vazquez, Fernando, Klimentidis, \& Sorensen, 2013; Makowsky et al., 2011) Polygenic (risk) scores (i.e. linear combinations of effect sizes for each SNP) are now commonly used to predict an individual's genetic liability to a disease or a trait. Consensus on polygenic scores as a standard metric, together with the availability of increasingly large dataset and refinement of analysis techniques (e.g. the introduction of genetic complex-trait analysis; see Yang et al., 2010; Yang, Lee, Goddard, \& Visscher, 2011), has enabled researchers to make significant progress in prediction of behavioral traits over the last decade. One notable example is educational achievement. While early GWAS-based models explained only around $2 \%$ of the variance (Rietveld et al., 2013), current models explain as much as $20 \%$ (Allegrini et al., 2019; Lee et al., 2018; Selzam et al. 2018; von Stumm et al., 2020). Similar improvements in levels of predictive performance can be observed for other complex phenotypes, such as personality (Power \& Pluess, 2015; Vinkhuyzen et al., 2012) and psychiatric traits (Schizophrenia Working Group of the Psychiatric Genomics Consortium, 2014; Visscher et al., 2012; Wray et al., 2014).

\section{Lexical norm datasets and megastudies in psycholinguistics}

Similar to behavioral genetics, psycholinguistics has witnessed a number of developments which have made it amenable to the introduction of evaluation benchmarks. The long-standing popularity of lexical decision paradigms has guaranteed a certain degree of methodological consensus, especially in research on lexical processing and semantics, and the use of large datasets has become common practice.

A number of large-scale lexical norms datasets have been developed and publicly shared (especially for En- glish and Dutch) over the last decades. The first significant effort in this direction was the MRC Psycholinguistic database (Coltheart, 1981), which gathered semantic, syntactic and lexical information for about 98,538 words, though many of these norms are now obsolete. More recent megastudies (Kessler, Treiman, \& Mullennix, 2002; Spieler \& Balota, 1997; Treiman, Mullennix, Bijeljac-Babic, \& Richmond-Welty, 1995; see http://crr.ugent.be/programs-data/megastudydata-available for a comprehensive list) have indexed increasingly large portions of the lexicon on wider sets of variables (Brysbaert \& New, Boris, 2009; Brysbaert, Stevens, De Deyne, Voorspoels, \& Storms, 2014; Chateau \& Jared, 2003; Kuperman, StadthagenGonzalez, \& Brysbaert, 2012; Tucker et al., 2019; Warriner, Kuperman, \& Brysbaert, 2013). Among the most notable examples is the English Lexicon Project dataset (Balota et al., 2007), which includes a wide range of psycholinguistic norms and reaction time data, gathered from a large participant sample, for around 40,000 words and around 40,000 non-words. More recently, datasets explicitly targeting psychologically and neurologically grounded features (Binder et al., 2016; Lynott, Connell, Brysbaert, Brand, \& Carney, 2020), and word association norms (De Deyne et al., 2019, 2016) have been developed and made publicly available.

Development of large-scale datasets has not only speeded up the transition to big data in psycholinguistics, but it has also contributed to a moving away from significance testing in favor of increased focus on model performance - though mostly still based on goodness-of-fit metrics. Furthermore, the use of largescale reference datasets has enabled convergence on a set of consensus predictive tasks of common interest and on the operationalization of relevant variables, which paves the way for the introduction of common evaluation benchmarks.

\section{Other potential applications}

Beyond these examples, there are many other domains where existing resources could be adapted into useful benchmarks, or where entirely new datasets could be acquired with this goal in mind. As a general heuristic, we expect that an emphasis on benchmark- 
ing will prove most productive in fields that have an applied focus, a robust methodological apparatus (solid constructs and consistent operationalizations), and/or agreement on core empirical problems and target metrics. However, even in fields that do not necessarily check these boxes, we believe that thinking about potentially useful benchmarks can draw much needed attention to the practical implications (or lack thereof) of existing research programs. To illustrate the wide range of potential applications, consider just a few examples drawn from diverse areas of psychology, and that vary considerably in feasibility and ambitiousness:

- In educational psychology, useful resources already exist that could be gathered and adapted to benchmarking purposes. For example, a number of international surveys are periodically conducted that target student outcomes at different ages and in a variety of domains, and that also gather information about student background, demographics, attitudes, home environment and school characteristics. Relevant resources include PISA (Program for International Student Assessment) reports, as well as data from PIRLS (Progress in International Reading Literacy Study), TIMMS (Trends in International Mathematics and Science Study) and PIAAC (Program for International Assessment of Adult Competencies) surveys. There is also considerable opportunity for development of valuable new datasets. Datasets pairing student outcomes with information on the size and network structure of classes, with duration and characteristics of live teaching, and with exposure to and type of online learning material could be used to experiment with and assess the efficacy of different pedagogical approaches, which could in turn inform attempts to optimize education curricula. MOOCs also offer a rich data source to tackle similar questions: course metadata (e.g. duration and type of video lectures, degree of interaction between students, amount of practical exercises and group work involved), automated annotations of the teaching material (e.g. quantification of linguistic styles and prosodic traits in videos), student feedback, and background information on learners' profiles could be collected to investigate which factors influence engagement and outcomes, and help optimize learning experiences.

- In personality, existing large-scale efforts to predict personality from social media data (e.g., Facebook likes or word use; see R. M. Brown, Roberts, \& Pollet, 2018; Park et al., 2015) could be used as a model for developing publicly available datasets that pair known personality scores with other behaviors. For example, a public dataset containing personality scores for tens of thousands of Reddit (https://reddit.com) users (shared with consent, of course) could provide an extremely powerful avenue for predictive challenges aimed at reconstructing personality from language use and online social interactions. Personality traits may also be reconstructed on the basis of metrics of individuals' standing in a social structure (number of followers/friends, position in one's friends' networks) or musical preferences (along the lines of Nave et al., 2018). Developing suitable open-access large-scale resources gathering this type of data would enable personality psychologists to investigate research questions which have hitherto been beyond reach for the academic community (and thus been the prerogative of corporate research).

- In clinical psychology, efforts are being made to standardize the measures and screening procedures administered to participants at intake of various studies, as well as the outcome measures administered to participants at fixed follow-up intervals. Competitive challenges could then be designed that incentivize researchers to develop predictive models that use the intake battery to predict changes in measures of global function (either collapsing across all treatments, or separately for specific interventions), attrition, or other clinically useful targets.

- In political psychology, social media datasets could be used to identify linguistic predictors of political affiliation (e.g. argumentative styles) and voting behavior; to model factors influencing 
the dynamics of key political phenomena such as the formation of echo chambers (along the lines of Baumann, Lorenz-Spreen, Sokolov, \& Starnini, 2020); to model and intervene on the emergence of polarization; or to identify the effect of framing strategies on attitudes towards rules and rule compliance. Political prediction markets are also a potentially interesting source of data which could be leveraged to evaluate models forecasting the outcome of key political events (Wijesinghe \& Rodrigues, 2012) and informing our understanding of what drives changes in political affiliation and beliefs.

- In social psychology, efforts have been made to develop large datasets that enable fine-grained modeling of social network structures alongside individuals' psychological traits and behavior. One example is the Copenhagen Networks Study, which involved tracking the location and digital interactions of a large cohort of students over an extended period of time, as well as profiling their personality and cognitive traits (see Sapiezynski, Stopczynski, Lassen, \& Lehmann, 2019). Releasing training sets where later time points are withheld for test purposes could enable researchers developing growth models to put their models to test with real-world challenges (e.g. predicting student dropout, or likelihood of seeking mental health help).

\section{Common pitfalls and concerns}

Benchmarks have the potential to revolutionize model evaluation practices in psychology in favor of higher reliability, objectivity and practical utility. They are not, however, exempt from potential drawbacks. Furthermore, the strong emphasis placed on predictive validity is likely to raise (more or less well-founded) concerns in the research community We will now review several caveats, potential pitfalls and common objections that deserve special attention, and provide suggestions on strategies to sidestep them wherever possible.

\section{Generalization is hard}

Some metrics are better than others in yielding trustworthy information on model performance. We have already highlighted how metrics that explicitly account for the risk of overfitting and value out-ofsample predictive accuracy tend to be more reliable than commonly used goodness-of-fit metrics. Still, no metric is perfect. The extent to which performance estimates from predictive metrics generalize to unseen data depends on characteristics of the dataset, even for robust metrics like cross-validation criteria. Small and/or non-representative samples often yield unreliable estimates. High predictive accuracy on data generated using a single experimental paradigm, for instance, may be an artifact of methodology-specific biases in the sample (e.g. systematic measurement error), and those accuracy levels may not generalize to samples collected using different methodologies. Metrics such as the generalization criterion (Busemeyer \& Wang, 2000) attempt to address this issue by imposing the constraint that validation sets should come from experimental designs different from those used in training. This is, however, only one of the many ways in which samples can fall short of being fully representative of the phenomenon or population of interest. Non-representative participant samples or stimulus sets may also affect generalizability to novel data.

\section{Goodhart's Law: when a measure becomes a target}

Specific benchmarks can prove extremely useful in certain stages of development of a discipline. But their limitations tend to emerge as soon as they become the primary or only criterion to evaluate model performance. As Goodhart's Law states, "when a measure becomes a target, it ceases to be a good measure". As the ImageNet vs. ObjectNet example discussed earlier illustrates, when optimizing for a specific task becomes the exclusive focus of model engineering efforts, researchers may lose sight of the fundamental goal of generating good predictions on other (e.g. real-world) instances of the task at stake. The development of good benchmarks should consequently always be viewed as an ongoing process. Tasks 
and metrics should be continuously (re-)evaluated and updated accordingly.

One way to mitigate Goodhart's Law is to develop multidimensional benchmarks - i.e. batteries of tasks models are jointly evaluated on. The concept of transfer learning in machine learning captures a similar principle -i.e. models trained to optimize performance on one set of tasks are evaluated based on their performance on multiple tasks (potentially including entirely new ones), without updating (or fine-tuning) parameters separately for each task (see McCann, Keskar, Xiong, \& Socher, 2018; Ruder, Peters, Swayamdipta, \& Wolf, 2019 for an example and an overview of transfer learning in natural language processing).

\section{Beyond predictive accuracy: validity and parsimony}

High predictive accuracy is, of course, not the only criterion models should be evaluated on in psychology, and our advocacy of benchmarking is not meant to discourage researchers from taking other criteria into account when designing or evaluating models Indeed, good predictive performance can sometimes be achieved by models that fail basic validity tests (J. I. Myung \& Pitt, 2018).

Criteria such as simplicity and parsimony are also often important considerations in the choice of model. There are several reasons for this. A sophisticated algorithm that performs well but is too complicated for a clinician to effectively apply in a real-world setting may be less useful than a "fast-and-frugal" heuristic that performs more poorly under optimal conditions (Gigerenzer \& Todd, 1999). And in cases where predictive performance is roughly comparable, simpler models are generally more desirable; fewer parameters reduce the risk of overfitting, and also the amount of compute required for training and deploying. Conversely, large models can easily become prohibitive in terms of computing resources; they come with large environmental costs (Strubell, Ganesh, \& McCallum, 2019); and their complexity may not be entirely justified in terms of performance gains. Some state-of-the-art transformer language models, for ex- ample, have proven to be overparameterized (Clark, Khandelwal, Levy, \& Manning, 2019; Kovaleva, Romanov, Rogers, \& Rumshisky, 2019): they contain many idle architectural units, and their performance levels are matched by smaller models trained using parsimonious schemes such as model distillation (e.g. Sanh, Debut, Chaumond, \& Wolf, 2019). It is thus important to incentivize researchers to strive for parsimonious solutions whenever possible - for example, by introducing the custom of quantifying and reporting complexity and resource requirements alongside any mention of predictive performance.

At the same time, an increased focus on benchmarking could potentially help discourage the somewhat common tendency to dismiss complex models out of hand merely because of their complexity, or on the basis of more subjective considerations. Qualitative criteria such as model plausibility, explanatory adequacy or faithfulness, which reflect the extent to which models are grounded in existing literature or in theoretical considerations, are often also advocated as central to model evaluation (I. J. Myung \& Pitt, 2002; J. I. Myung \& Pitt, 2018). However, the question of what role these criteria should play relative to clever model engineering is an open one. We lean towards a solution where qualitative constraints should in general not play a role in benchmark-based evaluation (e.g., model scores should not depend on a panel of judges' subjective assessment of theoretical elegance).

This does not mean that considerations like model complexity, computational efficiency, etc. cannot be explicitly factored into a model's score on a benchmark, but only that the model's score should be a deterministic function of clearly stated metrics, and should not depend on irreproducible components. Our position should also not be taken to imply that subjective considerations are unimportant in model evaluation; indeed, we think that a large element of subjectivity is probably unavoidable in psychology given the complexity and underdetermined nature of most psychological phenomena. But at the very least, researchers should strive to make it clear where objective metrics end and subjective valuations begin. 


\section{Interpretability}

As we have argued above, to achieve maximal reliability and utility, benchmark-based evaluation should rely on metrics that adequately capture the predictive validity (and, therefore, the generalizability) of a model. However, one particularly common objection to a push for greater emphasis on predictive accuracy is that models that yield higher predictive accuracy are intrinsically less interpretable than simpler (e.g. linear) ones, and thus fail to contribute to understanding the empirical phenomenon of interest.

It is true that, in many cases, the models yielding best performance are the more complex ones, as more parameters reduce prediction bias and offer the flexibility needed to capture complex patterns in the data. It is also true that parameters from complex models (e.g. weights in deep learning models) often lack a straightforward direct interpretation. However, the argument that higher complexity corresponds to lower interpretability - and for that matter, that interpretability is intrinsically desirable - builds on a number of questionable assumptions. Debunking these fallacies is especially important, as these arguments are among the reasons why complex models (e.g. deep learning models) are often "discarded for scientific purposes such as theory building and understanding" (Shmueli, 2010).

One of the assumptions behind the interpretability counterargument is that simpler (linear) models are more straightforwardly interpretable than complex (e.g. non-linear) ones. This statement is far from uncontroversial. Direct interpretability of individual parameter estimates in linear models is, in fact, highly dependent on overall properties of the model (i.e. covariates, degree of collinearity, interactions, etc.) and, though researchers in the explanatory tradition often tend to forget it, parameter estimates are always conditional on the model itself.

This point is nicely illustrated by recent neuroimaging and psychology studies (Botvinik-Nezer et al., 2020; Silberzahn et al., 2018) where independent research teams were asked to test a given set of hypotheses on the same dataset. Analytical approaches adopted by the teams can vary along a number of parameters (e.g. choice of covariates, statistical tests, coding procedures), resulting in quantitatively different feature coefficients and thus, potentially, in qualitatively different interpretations of the effect of such features. This variability in estimates cannot always be explained away by disparities in performance. Even models achieving comparable predictive accuracy can diverge widely in the importance attributed to individual features (Churchill, Yourganov, \& Strother, 2014; Schmah et al., 2010), regardless of the similarity between the models' structure or analytical form.

The flip side of this assumption is the idea that complex models are intrinsically uninterpretable, or, to put it more mildly, that they are much less interpretable than the cognitive processes they are meant to model. This common misconception likely stems from a certain ambiguity in the way interpretability is defined is the literature, but it does not ultimately stand up to scrutiny under any plausible definition (Lipton, 2018). If interpretability is defined in terms of transparency, i.e. as the possibility to directly $o b$ serve the sequence of algorithmic steps through which a decision/output has been produced, human cognitive processes are obviously no more interpretable than complex models (and probably much less so). If, on the other hand, interpretability is defined as the possibility to provide a post-hoc, compact natural language explanation of why a certain output was produced in response to a certain input, then humans and complex artificial models can, in principle, be equally interpretable. Saliency maps (Simonyan, Vedaldi, \& Zisserman, 2013), behavioral testing (Ribeiro, Wu, Guestrin, \& Singh, 2020), probing methods (Bolukbasi, Chang, Zou, Saligrama, \& Kalai, 2016; Bordia \& Bowman, 2019; Gardner et al., 2020; Kim et al., 2019; Linzen \& Baroni, 2020), and adversarial attacks (I. Goodfellow et al., 2014; I. J. Goodfellow, Shlens, \& Szegedy, 2014) are among the techniques that can be used to make the behavior of machine learning models more "explainable" (see Molnar, 2020 for an overview).

As these examples show, emphasis on predictive accuracy and, consequently, increased reliance on complex models in psychology need not result in a loss 
of interpretability. Similarly, it does not imply relinquishing ambitions of scientific understanding: even highly complex models can be probed and compared in ways which can inform mechanistic explanations of cognitive phenomena. Once again, machine learning offers some useful examples. A few studies have already shown how systematic comparison of benchmark performance of language models (e.g. Talmor, Elazar, Goldberg, \& Berant, 2019) which differ in the presence/absence and type of self-attention mechanism, or in the size of input context (long- vs. short-context models), can be used to corroborate theoretical intuitions on key aspects of human language processing. For instance, a recent study compared performance of different language model architectures in predicting psycholinguistic and neural language comprehension data (Schrimpf et al., 2020), showing that, while bidirectional attention models perform best on traditional NLP tasks, unidirectional language models predict fMRI data best. Recent work has more generally advocated for the development of cognitive benchmarks for language models (Artemova, Bakarov, Artemov, Burnaev, \& Sharaev, 2020; Hollenstein et al., 2019), which would enable comparative assessment of the cognitive plausibility of NLP models, while potentially shedding light on core principles of human language processing.

\section{What role for domain expertise?}

A final concern associated with an increased emphasis on benchmarking is that achieving good predictive performance on many tasks might turn out to require less traditional domain expertise than many researchers currently suppose. That is, it may turn out that the skills and knowledge bases needed to build good applied models in psychology have relatively little overlap with those emphasized in traditional psychological theorizing. This worry is not entirely without basis; it has been frequently observed that the winners of prediction-focused competitions, even when ostensibly focused on narrowly domainspecific problems, disproportionately tend to be teams of machine learning experts or data scientists with relatively little, and sometimes no, domain expertise (e.g., AlQuraishi, 2019; Bentzien, Muegge, Hamner,
\& Thompson, 2013).

We think that such a prospect - while perhaps uncomfortable in some ways - should if anything increase the utility of benchmarks in psychology. If it turned out that achieving good performance on major benchmarks in applied fields like educational psychology, psychopathology, and I-O psychology primarily requires computational rather than substantive expertise, this would constitute a powerful argument for emphasizing those skills to a greater extent in our training programs. To the degree that we believe that the public's investment in psychological science is ultimately aimed at improving the human condition, one might even argue that we have a collective moral obligation to subject the field to rigorous challenges of this kind - in much the same way that we have advocated for the objective evaluation of individual models.

\section{Conclusion}

Lack of consensus on robust metrics for model evaluation has long hindered progress in psychology. In this article, we have argued that introducing benchmarks can help overcome this fundamental issue, and we have provided general guidelines and concrete suggestions on how to go about developing metrics that suit the multifaceted profile of the field. Benchmarks inspired by real-world predictive challenges will not only provide psychology with communal evaluation metrics, but also motivate the community to redirect efforts towards solving problems with practical implications and high societal relevance.

\section{Acknowledgments}

This paper was supported by NIH awards R01MH096906 and R01MH109682. We are grateful to James D. Kent and Zachariah Marrero for comments, and to a group of participants at the 2018 meeting of the Society for Improvement of Psychological Science (SIPS) for discussion. 


\section{References}

Aliko, S., Huang, J., Gheorghiu, F., Meliss, S., \& Skipper, J. I. (2020). A'Naturalistic neuroimaging database'for understanding the brain using ecological stimuli. BioRxiv.

Allegrini, A. G., Selzam, S., Rimfeld, K., von Stumm, S., Pingault, J. B., \& Plomin, R. (2019, April). Genomic prediction of cognitive traits in childhood and adolescence. Mol. Psychiatry, 24(6), 819-827.

Allen, N. E., Sudlow, C., Peakman, T., Collins, R., \& UK Biobank. (2014, February). UK biobank data: come and get it. Sci. Transl. Med., $\underline{6}$ (224), $224 \mathrm{ed} 4$.

AlQuraishi, M. (2019, April). End-to-End differentiable learning of protein structure. Cell Syst, 8(4), 292-301.e3.

Artemova, E., Bakarov, A., Artemov, A., Burnaev, E., \& Sharaev, M. (2020, March). Data-driven models and computational tools for neurolinguistics: a language technology perspective.

Balota, D. A., Yap, M. J., Cortese, M. J., Hutchison, K. A., Kessler, B., Loftis, B., ... Treiman, R. (2007, August). The english lexicon project. Behav. Res. Methods, 39(3), 445-459.

Barbu, A., Mayo, D., Alverio, J., Luo, W., Wang, C., Gutfreund, D., ... Katz, B. (2019). ObjectNet: A large-scale bias-controlled dataset for pushing the limits of object recognition models. In H. Wallach, H. Larochelle, A. Beygelzimer, F. dAlché-Buc, E. Fox, \& R. Garnett (Eds.), Advances in neural information processing systems 32 (pp. 9453-9463). Curran Associates, Inc.

Baumann, F., Lorenz-Spreen, P., Sokolov, I. M., \& Starnini, M. (2020, January). Modeling echo chambers and polarization dynamics in social networks. Phys. Rev. Lett., 124(4), 048301.

Benjamin, C. F., Walshaw, P. D., Hale, K., Gaillard, W. D., Baxter, L. C., Berl, M. M., ... Bookheimer, S. Y. (2017, August). Presurgical language fMRI: Mapping of six critical regions. Hum. Brain Mapp., 38(8), 4239-4255.

Bennett, J., Lanning, S., \& Others. (2007). The netflix prize. In Proceedings of KDD cup and workshop (Vol. 2007, p. 35).

Bentzien, J., Muegge, I., Hamner, B., \& Thompson, D. C. (2013, May). Crowd computing: using competitive dynamics to develop and refine highly predictive models. Drug Discov. Today, 18(9-10), 472-478.

Binder, J. R., Conant, L. L., Humphries, C. J., Fernandino, L., Simons, S. B., Aguilar, M., \& Desai, R. H. (2016, May). Toward a brain-based componential semantic representation. Cogn. Neuropsychol., 33(3-4), 130-174.

Bolukbasi, T., Chang, K.-W., Zou, J. Y., Saligrama, V., \& Kalai, A. T. (2016). Man is to computer programmer as woman is to homemaker? debiasing word embeddings. In D. D. Lee, M. Sugiyama, U. V. Luxburg, I. Guyon, \& R. Garnett (Eds.), Advances in neural information processing systems 29 (pp. 4349-4357). Curran Associates, Inc.

Bookheimer, S. (2007, June). Pre-surgical language mapping with functional magnetic resonance imaging. Neuropsychol. Rev., 17(2), 145-155.

Border, R., Johnson, E. C., Evans, L. M., Smolen, A., Berley, N., Sullivan, P. F., \& Keller, M. C. (2019, May). No support for historical candidate gene or candidate Gene-by-Interaction hypotheses for major depression across multiple large samples. Am. J. Psychiatry, 176(5), 376-387.

Bordia, S., \& Bowman, S. R. (2019, April). Identifying and reducing gender bias in Word-Level language models.

Botvinik-Nezer, R., Holzmeister, F., Camerer, C. F., Dreber, A., Huber, J., Johannesson, M., ... Schonberg, T. (2020, June). Variability in the analysis of a single neuroimaging dataset by many teams. Nature, 582(7810), 84-88.

Breiman, L. (2001, August) . Statistical modeling: The two cultures (with comments and a rejoinder by the author). Stat. Sci., 16(3), 199-231.

Brown, R. M., Roberts, S. G. B., \& Pollet, T. V. (2018, August). HEXACO personality factors and their associations with facebook use and facebook network characteristics.

Brown, T. B., Mann, B., Ryder, N., Subbiah, M., Kaplan, J., Dhariwal, P., ... Amodei, D. (2020, May). Language models are Few-Shot learners. 
Brysbaert, M., \& New, Boris. (2009, November). Moving beyond kučera and francis: A critical evaluation of current word frequency norms and the introduction of a new and improved word frequency measure for american english. Behav. Res. Methods, 41(4), 977-990.

Brysbaert, M., Stevens, M., De Deyne, S., Voorspoels, W., \& Storms, G. (2014, July). Norms of age of acquisition and concreteness for 30,000 dutch words. Acta Psychol., 150, 80-84.

Busemeyer, J. R., \& Wang, Y. M. (2000, March). Model comparisons and model selections based on generalization criterion methodology. J. Math. Psychol., 44(1), 171-189.

Button, K. S., Ioannidis, J. P. A., Mokrysz, C., Nosek, B. A., Flint, J., Robinson, E. S. J., \& Munafò, M. R. (2013, May). Power failure: why small sample size undermines the reliability of neuroscience. Nat. Rev. Neurosci., 14(5), 365-376.

Chabris, C. F., Lee, J. J., Cesarini, D., Benjamin, D. J., \& Laibson, D. I. (2015, July). The fourth law of behavior genetics. Curr. Dir. Psychol. Sci., 24(4), 304-312.

Chateau, D., \& Jared, D. (2003, February). Spellingsound consistency effects in disyllabic word naming. J. Mem. Lang., 48(2), 255-280.

Chavan, V., \& Penev, L. (2011). The data paper: a mechanism to incentivize data publishing in biodiversity science. BMC bioinformatics, 12(S15), S2.

Chen, T., Kornblith, S., Norouzi, M., \& Hinton, G. (2020, February). A simple framework for contrastive learning of visual representations.

Churchill, N. W., Yourganov, G., \& Strother, S. C. (2014). Comparing within-subject classification and regularization methods in fMRI for large and small sample sizes. Hum. Brain Mapp., 35(9), 4499-4517.

Clark, K., Khandelwal, U., Levy, O., \& Manning, C. D. (2019). What does BERT look at? an analysis of BERT's attention.

Coltheart, M. (1981, November). The MRC psycholinguistic database. The Quarterly Journal of Experimental Psychology Section A, 33(4), 497-505.

Cronbach, L. J., \& Meehl, P. E. (1955, July). Con- struct validity in psychological tests. Psychol. Bull., 52(4), 281-302.

Cumming, G. (2013). Understanding the new statistics: Effect sizes, confidence intervals, and Meta-Analysis. Routledge.

Danielsen, A. A., Fenger, M. H. J., Østergaard, S. D., Nielbo, K. L., \& Mors, O. (2019, August). Predicting mechanical restraint of psychiatric inpatients by applying machine learning on electronic health data. Acta Psychiatr. Scand., 140(2), 147-157.

De Deyne, S., Navarro, D. J., Perfors, A., Brysbaert, M., \& Storms, G. (2019, June). The "small world of words" english word association norms for over 12,000 cue words. Behav. Res. Methods, 51(3), 987-1006.

De Deyne, S., Perfors, A., \& Navarro, D. J. (2016). Predicting human similarity judgments with distributional models: The value of word associations. In Proceedings of COLING 2016, the 26th international conference on computational linguistics: Technical papers (pp. 1861-1870). de Los Campos, G., Sorensen, D., \& Gianola, D. (2015, May). Genomic heritability: what is it? PLoS Genet., 11(5), e1005048.

de Los Campos, G., Vazquez, A. I., Fernando, R., Klimentidis, Y. C., \& Sorensen, D. (2013, July). Prediction of complex human traits using the genomic best linear unbiased predictor. PLoS Genet., 9 (7), e1003608.

Deng, J., Dong, W., Socher, R., Li, L., Kai Li, \& Li Fei-Fei. (2009, June). ImageNet: A large-scale hierarchical image database. In 2009 IEEE conference on computer vision and pattern recognition (pp. 248-255).

Devlin, J., Chang, M.-W., Lee, K., \& Toutanova, K. (2018, October). BERT: Pre-training of deep bidirectional transformers for language understanding.

Dudbridge, F. (2013, March). Power and predictive accuracy of polygenic risk scores. PLoS Genet., $9(3)$, e1003348.

Duncan, L. E., \& Keller, M. C. (2011, October). A critical review of the first 10 years of candidate gene-by-environment interaction research in psychiatry. Am. J. Psychiatry, 168(10), 1041-1049. 
Eisenberg, I. W., Bissett, P. G., Zeynep Enkavi, A., Li, J., MacKinnon, D. P., Marsch, L. A., \& Poldrack, R. A. (2019, May). Uncovering the structure of self-regulation through data-driven ontology discovery. Nat. Commun., 10(1), 2319.

Ferguson, C. J., \& Heene, M. (2012, November). A vast graveyard of undead theories: Publication bias and psychological science's aversion to the null. Perspect. Psychol. Sci., 7(6), 555-561.

Fisher, R. A. (1919). XV.-The correlation between relatives on the supposition of mendelian inheritance. Earth Environ. Sci. Trans. R. Soc. Edinb., 52(2), 399-433.

Frank, M. C., Bergelson, E., Bergmann, C., Cristia, A., Floccia, C., Gervain, J., ... Others (2017). A collaborative approach to infant research: Promoting reproducibility, best practices, and theory-building. Infancy, 22(4), 421-435.

Garcia Martinez, M., \& Walton, B. (2014, April). The wisdom of crowds: The potential of online communities as a tool for data analysis Technovation, 34(4), 203-214.

Gardner, M., Artzi, Y., Basmova, V., Berant, J., Bogin, B., Chen, S., ... Zhou, B. (2020, April). Evaluating NLP models via contrast sets.

Gardner, M., Grus, J., Neumann, M., Tafjord, O., Dasigi, P., Liu, N., ... Zettlemoyer, L. (2018, March). AllenNLP: A deep semantic natural language processing platform.

Gelman, A. (2013). Commentary: P values and statistical practice. Epidemiology, 24(1), 69-72.

Gelman, A., \& Weakliem, D. (2009). Of beauty, sex and power: Too little attention has been paid to the statistical challenges in estimating small effects. Am. Sci., 97(4), 310-316.

Gigerenzer, G., \& Todd, P. M. (1999). Fast and frugal heuristics: The adaptive toolbox. In Simple heuristics that make us smart (pp. 3-34). Oxford University Press.

Goodfellow, I., Pouget-Abadie, J., Mirza, M., Xu, B., Warde-Farley, D., Ozair, S., ... Bengio, Y. (2014). Generative adversarial nets. In Z. Ghahramani, M. Welling, C. Cortes, N. D. Lawrence, \& K. Q. Weinberger (Eds.), Advances in neural information processing systems 27 (pp. 2672-2680). Curran Associates, Inc.
Goodfellow, I. J., Shlens, J., \& Szegedy, C. (2014, December). Explaining and harnessing adversarial examples.

Gorgolewski, K., Esteban, O., Schaefer, G., Wandell, B., \& Poldrack, R. (2017). OpenNeuro-a free online platform for sharing and analysis of neuroimaging data. Organization for human brain mapping. Vancouver, Canada, 1677(2).

Gorgolewski, K., Margulies, D. S., \& Milham, M. P. (2013). Making data sharing count: a publication-based solution. Frontiers in neuroscience, 7,9 .

Gorgolewski, K. J., Auer, T., Calhoun, V. D., Craddock, R. C., Das, S., Duff, E. P., ... Poldrack, R. A. (2016, June). The brain imaging data structure, a format for organizing and describing outputs of neuroimaging experiments. Sci Data, 3, 160044 .

Greenland, S. (2011, October). Null misinterpretation in statistical testing and its impact on health risk assessment. Prev. Med., 53(4-5), 225-228.

Guzzo, R. A., Fink, A. A., King, E., Tonidandel, S., \& Landis, R. S. (2015, December). Big data recommendations for Industrial-Organizational psychology. Ind. Organ. Psychol., 8(4), 491-508.

Haworth, C. M. A., Davis, O. S. P., \& Plomin, R. (2013, February). Twins early development study (TEDS): a genetically sensitive investigation of cognitive and behavioral development from childhood to young adulthood. Twin Res. Hum. Genet., 16(1), 117-125.

Hofman, J. M., Sharma, A., \& Watts, D. J. (2017, February). Prediction and explanation in social systems. Science, 355(6324), 486-488.

Hollenstein, N., Barrett, M., Troendle, M., Bigiolli, F., Langer, N., \& Zhang, C. (2019, April). Advancing NLP with cognitive language processing signals.

Hur, Y.-M., \& Craig, J. M. (2013, February). Twin registries worldwide: an important resource for scientific research. Twin Res. Hum. Genet., 16(1), $1-12$.

Ioannidis, J. P. A. (2008, September). Why most discovered true associations are inflated. Epidemiology, 19(5), 640-648.

Jacowitz, K. E., \& Kahneman, D. (1995, November). 
Measures of anchoring in estimation tasks. Pers. Soc. Psychol. Bull., 21(11), 1161-1166.

Kahneman, D. (1965, November). Control of spurious association and the reliability of the controlled variable. Psychol. Bull., 64(5), 326-329.

Kalluri, P. (2020, July). Don't ask if artificial intelligence is good or fair, ask how it shifts power. Nature, 583(7815), 169.

Kessler, B., Treiman, R., \& Mullennix, J. (2002, July). Phonetic biases in voice key response time measurements. J. Mem. Lang., 47(1), 145-171.

Kim, N., Patel, R., Poliak, A., Wang, A., Xia, P., Thomas McCoy, R., ... Pavlick, E. (2019, April). Probing what different NLP tasks teach machines about function word comprehension.

Klein, R. A., Ratliff, K. A., Vianello, M., Adams, R. B., Bahník, Š., Bernstein, M. J., ... Nosek, B. A. (2014, May). Investigating variation in replicability. Soc. Psychol., 45(3), 142-152.

Kline, R. B. (2013). Beyond significance testing: Statistics reform in the behavioral sciences. American Psychological Association.

Kovaleva, O., Romanov, A., Rogers, A., \& Rumshisky, A. (2019, August). Revealing the dark secrets of BERT.

Kozlowski, S. W. J., \& Chao, G. T. (2018, May). Unpacking team process dynamics and emergent phenomena: Challenges, conceptual advances, and innovative methods. Am. Psychol., 73(4), $576-592$.

Krizhevsky, A., Sutskever, I., \& Hinton, G. E. (2012). ImageNet classification with deep convolutional neural networks. In F. Pereira, C. J. C. Burges, L. Bottou, \& K. Q. Weinberger (Eds.), Advances in neural information processing systems 25 (pp. 1097-1105). Curran Associates, Inc.

Kuperman, V., Stadthagen-Gonzalez, H., \& Brysbaert, M. (2012, December). Age-of-acquisition ratings for 30,000 english words. Behav. Res. Methods, 44(4), 978-990.

LeCun, Y., Bengio, Y., \& Hinton, G. (2015, May). Deep learning. Nature, 521(7553), 436-444.

Lee, J. J., Wedow, R., Okbay, A., Kong, E., Maghzian, O., Zacher, M., ... Others (2018). Gene discovery and polygenic prediction from a 1.1-millionperson GWAS of educational attainment. Nat.
Genet., 50(8), 1112.

Linzen, T., \& Baroni, M. (2020, April). Syntactic structure from deep learning.

Lynott, D., Connell, L., Brysbaert, M., Brand, J., \& Carney, J. (2020, June). The lancaster sensorimotor norms: multidimensional measures of perceptual and action strength for 40,000 english words. Behav. Res. Methods, 52(3), 1271-1291.

Mahmoodi, J., Leckelt, M., van Zalk, M. W. H., Geukes, K., \& Back. (2017, December). Big data approaches in social and behavioral science: four key trade-offs and a call for integration. Current Opinion in Behavioral Sciences, 18, 57-62.

Makowsky, R., Pajewski, N. M., Klimentidis, Y. C., Vazquez, A. I., Duarte, C. W., Allison, D. B., \& de los Campos, G. (2011, April). Beyond missing heritability: prediction of complex traits. PLoS Genet., 7(4), e1002051.

McCann, B., Keskar, N. S., Xiong, C., \& Socher, R. (2018, June). The natural language decathlon: Multitask learning as question answering.

McShane, B. B., Gal, D., Gelman, A., Robert, C., \& Tackett, J. L. (2019, March). Abandon statistical significance. null, 73(sup1), 235-245.

Meehl, P. E. (1978). Theoretical risks and tabular asterisks: Sir karl, sir ronald, and the slow progress of soft psychology. J. Consult. Clin. Psychol., 46(4), 806.

Meehl, P. E. (1990, February). Why summaries of research on psychological theories are often uninterpretable. Psychol. Rep., 66(1), 195-244.

Miller, G. A., Beckwith, R., Fellbaum, C., Gross, D., \& Miller, K. J. (1990). Introduction to WordNet: An on-line lexical database. International journal of lexicography, 3(4), 235-244.

Mischel, W. (2008, December). The toothbrush problem. https:// $\overline{\text { www }}$ .psychologicalscience.org/observer/ the-toothbrush-problem. (Accessed: 2020-918)

Molnar, C. (2020). Interpretable machine learning. Lulu.com.

Moshontz, H., Campbell, L., Ebersole, C. R., IJzerman, H., Urry, H. L., Forscher, P. S., ... Chartier, C. R. (2018, December). The psychological science accelerator: Advancing psychol- 
ogy through a distributed collaborative network. Adv Methods Pract Psychol Sci, 1(4), 501-515.

Mosier, C. I. (1951, April). I. problems and designs of Cross-Validation 1. Educ. Psychol. Meas., 11(1), 5-11.

Myung, I. J., \& Pitt, M. A. (2002). Mathematical modeling.

Myung, J. I., \& Pitt, M. A. (2018). Model comparison in psychology. Stevens' Handbook of Experimental Psychology and Cognitive Neuroscience, 5, 1-34.

Nakagawa, S., \& Cuthill, I. C. (2007, November). Effect size, confidence interval and statistical significance: a practical guide for biologists. Biol. Rev. Camb. Philos. Soc., 82(4), 591-605.

Nastase, S. A., Liu, Y. F., Hillman, H., Zadbood, A., Hasenfratz, L., Keshavarzian, N., ... Others (2019). Narratives: fMRI data for evaluating models of naturalistic language comprehension. OpenNeuro https://doi. org/10. 18112/openneuro. ds002345. v1. 0. 1.

Nave, G., Minxha, J., Greenberg, D. M., Kosinski, M., Stillwell, D., \& Rentfrow, J. (2018, July). Musical preferences predict personality: Evidence from active listening and facebook likes. Psychol. Sci., 29(7), 1145-1158.

Oberauer, K., Lewandowsky, S., Awh, E., Brown, G. D. A., Conway, A., Cowan, N., ... Ward, G. (2018, September). Benchmarks for models of short-term and working memory. Psychol. Bull., 144(9), 885-958.

Open Science Collaboration. (2015, August). Estimating the reproducibility of psychological science. Science, 349(6251), aac4716.

Park, G., Schwartz, H. A., Eichstaedt, J. C., Kern, M. L., Kosinski, M., Stillwell, D. J., ... Seligman, M. E. P. (2015, June). Automatic personality assessment through social media language. J. Pers. Soc. Psychol., 108(6), 934-952.

Plomin, R., DeFries, J. C., Knopik, V. S., \& Neiderhiser, J. M. (2016, January). Top 10 replicated findings from behavioral genetics. Perspect. Psychol. Sci., 11(1), 3-23.

Power, R. A., \& Pluess, M. (2015, July). Heritability estimates of the big five personality traits based on common genetic variants. Transl. Psychiatry,
5, e604.

Ribeiro, M. T., Singh, S., \& Guestrin, C. (2016, June). Model-Agnostic interpretability of machine learning.

Ribeiro, M. T., Wu, T., Guestrin, C., \& Singh, S. (2020). Beyond accuracy: Behavioral testing of NLP models with CheckList.

Rietveld, C. A., Medland, S. E., Derringer, J., Yang, J., Esko, T., Martin, N. W., ... Koellinger, P. D. (2013, June). GWAS of 126,559 individuals identifies genetic variants associated with educational attainment. Science, 340(6139), 1467-1471.

Rohrer, J. M. (2018). Thinking clearly about correlations and causation: Graphical causal models for observational data. Advances in Methods and Practices in.

Ruder, S., Peters, M. E., Swayamdipta, S., \& Wolf, T. (2019). Transfer learning in natural language processing.

Sakaguchi, K., Le Bras, R., Bhagavatula, C., \& Choi, Y. (2019, July). WinoGrande: An adversarial winograd schema challenge at scale.

Sanh, V., Debut, L., Chaumond, J., \& Wolf, T. (2019). DistilBERT, a distilled version of BERT: smaller, faster, cheaper and lighter. arXiv preprint arXiv:1910. 01108.

Sapiezynski, P., Stopczynski, A., Lassen, D. D., \& Lehmann, S. (2019, December). Interaction data from the copenhagen networks study. Sci Data, $\underline{6}(1), 315$.

Schizophrenia Working Group of the Psychiatric Genomics Consortium. (2014, July). Biological insights from 108 schizophrenia-associated genetic loci. Nature, 511(7510), 421-427.

Schmah, T., Yourganov, G., Zemel, R. S., Hinton, G. E., Small, S. L., \& Strother, S. C. (2010, November). Comparing classification methods for longitudinal fMRI studies. Neural Comput., 22(11), 2729-2762.

Schrimpf, M., Blank, I. A., Tuckute, G., Kauf, C., Hosseini, E. A., \& others. (2020). Artificial neural networks accurately predict language processing in the brain. BioRxiv.

Selzam, S., Krapohl, E., von Stumm, S., O'Reilly, P. F., Rimfeld, K., Kovas, Y., ... Plomin, R. 
(2018, January). Predicting educational achievement from DNA. Mol. Psychiatry, 23(1), 161.

Shear, B. R., \& Zumbo, B. D. (2013, October). False positives in multiple regression: Unanticipated consequences of measurement error in the predictor variables. Educ. Psychol. Meas., 73(5), $733-756$.

Shmueli, G. (2010). To explain or to predict? Stat. Sci..

Shridhar, M., Thomason, J., Gordon, D., Bisk, Y., Han, W., Mottaghi, R., ... Fox, D. (2020). Alfred: A benchmark for interpreting grounded instructions for everyday tasks. In Proceedings of the IEEE/CVF conference on computer vision and pattern recognition (pp. 10740-10749).

Silberzahn, R., Uhlmann, E. L., Martin, D. P., Anselmi, P., Aust, F., Awtrey, E., ... Nosek, B. A. (2018, September). Many analysts, one data set: Making transparent how variations in analytic choices affect results. Advances in Methods and Practices in Psychological Science, 1(3), $337-356$.

Simonyan, K., Vedaldi, A., \& Zisserman, A. (2013, December). Deep inside convolutional networks: Visualising image classification models and saliency maps.

Spieler, D. H., \& Balota, D. A. (1997, November). Bringing computational models of word naming down to the item level. Psychol. Sci., $\underline{8}(6)$, 411-416.

Strubell, E., Ganesh, A., \& McCallum, A. (2019). Energy and policy considerations for deep learning in NLP.

Sullivan, G. M., \& Feinn, R. (2012, September). Using effect size - or why the $\mathrm{P}$ value is not enough. J. Grad. Med. Educ., 4(3), 279-282.

Talmor, A., Elazar, Y., Goldberg, Y., \& Berant, J. (2019, December). oLMpics - on what language model pre-training captures.

Treiman, R., Mullennix, J., Bijeljac-Babic, R., \& Richmond-Welty, E. D. (1995, June). The special role of rimes in the description, use, and acquisition of english orthography. J. Exp. Psychol. Gen., 124(2), 107-136.

Tucker, B. V., Brenner, D., Danielson, D. K., Kelley, M. C., Nenadić, F., \& Sims, M. (2019,
June). The massive auditory lexical decision (MALD) database. Behav. Res. Methods, 51(3), 1187-1204.

Van Essen, D. C., Smith, S. M., Barch, D. M., Behrens, T. E. J., Yacoub, E., Ugurbil, K., \& WU-Minn HCP Consortium. (2013, October). The WUMinn human connectome project: an overview. Neuroimage, 80, 62-79.

Vasishth, S., Mertzen, D., Jäger, L. A., \& Gelman, A. (2018). The statistical significance filter leads to overoptimistic expectations of replicability (Vol. 103).

Vaswani, A., Shazeer, N., Parmar, N., Uszkoreit, J., Jones, L., Gomez, A. N., ... Polosukhin, I. (2017). Attention is all you need. In I. Guyon et al. (Eds.), Advances in neural information processing systems 30 (pp. 5998-6008). Curran Associates, Inc.

Vinkhuyzen, A. A. E., Pedersen, N. L., Yang, J., Lee, S. H., Magnusson, P. K. E., Iacono, W. G., ... Wray, N. R. (2012, April). Common SNPs explain some of the variation in the personality dimensions of neuroticism and extraversion. Transl. Psychiatry, 2, e102.

Visscher, P. M., Brown, M. A., McCarthy, M. I., \& Yang, J. (2012, January). Five years of GWAS discovery. Am. J. Hum. Genet., 90(1), 7-24.

von Stumm, S., Smith-Woolley, E., Ayorech, Z., McMillan, A., Rimfeld, K., Dale, P. S., \& Plomin, R. (2020, May). Predicting educational achievement from genomic measures and socioeconomic status. Dev. Sci., 23(3), e12925.

Wang, A., Pruksachatkun, Y., Nangia, N., Singh, A., Michael, J., Hill, F., ... Bowman, S. (2019). SuperGLUE: A stickier benchmark for General-Purpose language understanding systems. In H. Wallach, H. Larochelle, A. Beygelzimer, F. dAlché-Buc, E. Fox, \& R. Garnett (Eds.), Advances in neural information processing systems 32 (pp. 3266-3280). Curran Associates, Inc.

Warriner, A. B., Kuperman, V., \& Brysbaert, M. (2013, December). Norms of valence, arousal, and dominance for 13,915 english lemmas. Behav. Res. Methods, 45(4), 1191-1207.

Watts, D. J. (2014, September). Common sense 
and sociological explanations. AJS, 120(2), 313-351.

Watts, D. J., Beck, E. D., Bienenstock, E. J., Bowers, J., Frank, A., \& others. (n.d.). Explanation, prediction, and causality: Three sides of the same coin? osf.io.

Westfall, J., \& Yarkoni, T. (2016, March). Statistically controlling for confounding constructs is harder than you think. PLoS One, 11(3), e0152719.

Wijesinghe, S., \& Rodrigues, J. (2012). Political prediction markets: Can we use them to predict election outcomes?

Wray, N. R., Lee, S. H., Mehta, D., Vinkhuyzen, A. A. E., Dudbridge, F., \& Middeldorp, C. M. (2014, October). Research review: Polygenic methods and their application to psychiatric traits. J. Child Psychol. Psychiatry, 55(10), 1068-1087.

Wright, S. (1920, June). The relative importance of heredity and environment in determining the piebald pattern of Guinea-Pigs. Proc. Natl. Acad. Sci. U. S. A., 6(6), 320-332.

Yang, J., Benyamin, B., McEvoy, B. P., Gordon, S., Henders, A. K., Nyholt, D. R., ... Visscher, P. M. (2010, July). Common SNPs explain a large proportion of the heritability for human height. Nat. Genet., 42(7), 565-569.

Yang, J., Lee, S. H., Goddard, M. E., \& Visscher, P. M. (2011, January). GCTA: a tool for genome-wide complex trait analysis. Am. J. Hum. Genet., 88(1), 76-82.

Yarkoni, T. (2009, May). Big correlations in little studies: Inflated fMRI correlations reflect low statistical Power-Commentary on vul et al. (2009). Perspect. Psychol. Sci., 4(3), 294-298.

Yarkoni, T. (2019, November). The generalizability crisis.

Yarkoni, T., Markiewicz, C. J., de la Vega, A., Gorgolewski, K. J., Salo, T., Halchenko, Y. O., ... Blair, R. (2019, August). PyBIDS: Python tools for BIDS datasets. J Open Source Softw, 4 (40).

Yarkoni, T., \& Westfall, J. (2017, November). Choosing prediction over explanation in psychology: Lessons from machine learning. Perspect. Psychol. Sci., 12(6), 1100-1122.

Zou, J., \& Schiebinger, L. (2018, July). AI can be sexist and racist - it's time to make it fair. Nature, 559(7714), 324-326. 